\title{
CHÉTOTAXIES CERCARIENNES COMPARÉES DE DIX ESPËCES DE SCHISTOSOMES
}

\author{
Ch. BAYSSADE-DUFOUR*
}

RÉSUMÉ. La chétotaxie cercarienne permet de différencier quatre groupes de Schistosomatinae :

- Schistosomatium douthitti, parasite de Stagnicola et Lymnaea (Lymnaeidae) ;

- Schistosoma japonicum, parasite d'Oncomelania (Melaniidae) ;

- Schistosoma mansoni et $S$. rodhaini, parasites de Biomphalaria (Planorbiinae, Bulinidae) ;

- Schistosoma haematobium, S. bovis, S. margrebosviei, S. mattheei, S. leiperi et S. intercalatum, parasites d'Isidora, Physopsis et Bulinus (Bulininae, Bulinidae).

Dans le troisième groupe, elle permet l'identification de chacune des deux espèces : $S$. mansoni et $S$. rodhaini.

Dans le quatrième groupe, elle montre de petites différences statistiques dans la topographie des sensilles de chacune des six espèces considérées.

\section{Comparison of cercarial chaetotaxy of ten species of Schistosomes.}

SUMMARY. The cercarial chaetotaxy permits the differenciation of four groups of Schistosomatinae.

- Schistosomatium douthitti, parasite of Stagnicola and Lymnaea (Lymnaeidae);

- Schistosoma japonicum, parasite of Oncomelania (Melaniidae);

- Schistosoma mansoni and S. rodhaini, parasites of Biomphalaria (Planorbiinae, Bulinidae);

- Schistosoma haematobium, S. bovis, S. margrebosviei, S. mattheei, S. leiperi and S. intercalatum, parasites of Isidora, Physopsis and Bulinus (Bulininae, Bulinidae).

For the third group, cercaria of Schistosoma mansoni and S. rodhaini may be distinguished.

In the fourth group, small statistical differences in the topography of the sensillae can be shown between the various species.

Nous disposons, soit par les données bibliographiques, soit par des préparations originales, de renseignements précis sur la chétotaxie cercarienne d'une trentaine de souches représentant dix espèces de Schistosomes appartenant à deux genres : Schistosomatium et Schistosoma; il s'agit de Schistosomatium douthitti, Schistosoma japonicum, S. mansoni, S. rodhaini, S. haematobium, S. bovis, S. margrebowiei, S. mattheei, S. leiperi et S. intercalatum.

\footnotetext{
* Laboratoire de Zoologie des Vers, associé au CNRS, Muséum National d'Histoire Naturelle,
} 61, rue Buffon, F 75231 Paris Cedex 05

Accepté le 23 février 1982. 
Les différences constatées sont parfois très nettes, parfois légères et d'ordre statistique et nous n'en connaissons pas alors l'exacte signification mais elle paraissent utiles à signaler car elles peuvent se révéler caractéristiques de races géographiques, comme nous l'avons observé chez Schistosoma mansoni africain, statistiquement différent de S. mansoni américain; cf Bayssade-Dufour (1977a)

\section{I - Origine des documents utilisés.}

\section{Schistosomatium douthitti}

La chétotaxie de la cercaire a fait lobjet de deux descriptions : par Wagner (1961) et par Knos et Short (1979).

Wagner décrit une souche originaire du Michigan (États-Unis d'Amérique) entretenue en laboratoire sur Lymnaea palustris. Une cercaire entière est dessinée en vue ventrale et dorsale. Knos et Short décrivent une souche également originaire du Michigan, entretenue en laboratoire sur Souris blanche et deux genres de Lymnaeidae : Stagnicola emarginata angulata et Lymnaea palustris. Les dessins des papilles céphaliques et caudales tiennent compte d'observations faites sur 20 cercaires provenant de l'un et l'autre Mollusque ; ceux des papilles corporelles, d'observations faites sur 40 cercaires, provenant, elles aussi, de l'un et l'autre Mollusque. Les auteurs ne signalent aucune différence susceptible d'être liée à l'espèce du Mollusque.

\section{Schistosoma japonicum}

La chétotaxie de la cercaire est décrite par Sakamoto et Ishii (1978) et par Albàret et coll. (1982).

Sakamoto et Ishii décrivent une souche entretenue sur Oncomelania nosophora et Lapin. Une cercaire entière est dessinée en vue ventrale, dorsale et latérale ; une photographie, prise au microscope à balayage, montre les papilles $\mathrm{C}_{\mathrm{I}} \mathrm{L}$ et les orifices des glandes de pénétration.

Albaret et coll. décrivent deux souches, passant respectivement sur Oncomelania hupensis hupensis, O. h. nosophora et Souris blanche.

\section{Schistosoma mansoni}

La chétotaxie de la cercaire est donnée par Vercammen-Grandjean (1951), Wagner (1961), Richard (1968 et 1971), Short et Cartrett (1973), Short et Kuntz (1976) et par Bayssade-Dufour (1977a, b, 1979) ; nous disposons donc d'un abondant matériel constitué de souches africaines et américaines, passant par Biomphalaria pfeifferi, $B$. alexandrina, $B$. sudanica ou $B$. glabrata, provenant d'hôtes vertébrés naturels : Homme, Rattus rattus, ou expérimentaux : Singes Erythrocebus patas, Souris blanches ou Hamsters. 


\section{Schistosoma rodhaini}

La cercaire est décrite par Fripp (1967), Richard (1968 et 1971), Short et Kuntz (1976) et par nous-même dans le présent travail. Fripp, Richard, Short et Kuntz décrivent des souches d'Ouganda, Kenya et Afrique du Sud parasites de Biomphalaria sudanica tanganyicensis $B$. pfeifferi nairobiensis, B. sudanica, B. glabrata et dont les hôtes vertébrés sont des Souris blanches, Hamsters, Lapins et Cobayes. Nous décrivons une souche du Kenya parasite de B. pfeifferi et Praomys natalensis.

\section{Schistosoma haematobium}

Décrit par Richard (1968 et 1971) et par nous-même dans ce travail. Richard décrit une souche d'origine marocaine, entretenue en laboratoire sur Isidora truncata et Mesocricetus auratus. Nous décrivons une souche humaine de République Centrafricaine passant par Isidora rohlfsi.

\section{Schistosoma bovis}

Décrit par Richard (1968 et 1971) d'après une souche de Sardaigne (Italie), parasite d'Isidora truncata et Mouton.

\section{Schistosoma margrebowiei}

Décrit dans ce travail, d'après une souche du Botswana, parasite d'Isidora tropica et Mesocricetus auratus.

\section{Schistosoma mattheei}

Décrit dans ce travail, d'après une souche d'Afrique du Sud, parasite de Physopsis sp. et Praomys natalensis.

\section{Schistosoma leiperi}

Décrit dans ce travail, d'après une souche du Botswana, parasite de Physopsis sp. et Praomys natalensis.

\section{Schistosoma intercalatum}

Décrit par Bayssade-Dufour et coll. (1980) et par nous-même dans ce travail. Bayssade-Dufour et coll. décrivent une souche humaine du Gabon passant par Bulinus forskalii. Nous décrivons une seconde souche humaine du Gabon, obtenue à partir d'un autre malade et passant aussi par Bulinus forskalii.

\section{II - Séparation des Schistosomes en fonction de la famille ou sous-famille de Mollusques parasités.}

Ces différents Schistosomes ont des caractéristiques chétotaxiques qui permettent la séparation en quatre groupes : les Schistosomes de Lymnaeidae, de Melaniidae, de Planorbiinae et de Bulininae. 


\section{1) Caractéristiques de Schistosomatium douthitti parasite de Lymnaeidae} (Fig. $1 \mathrm{~A}, 2 \mathrm{D}, 3 \mathrm{D}, 4 \mathrm{C}$ )

La cercaire de Schistosomatium douthitti présente des caractères différentiels, portés au niveau des papilles céphaliques, corporelles ventrales et dorsales, acétabulaires, et caudales ventrales et dorsales, qui l'opposent à ceux des neuf espèces de Schistosoma que nous connaissons :
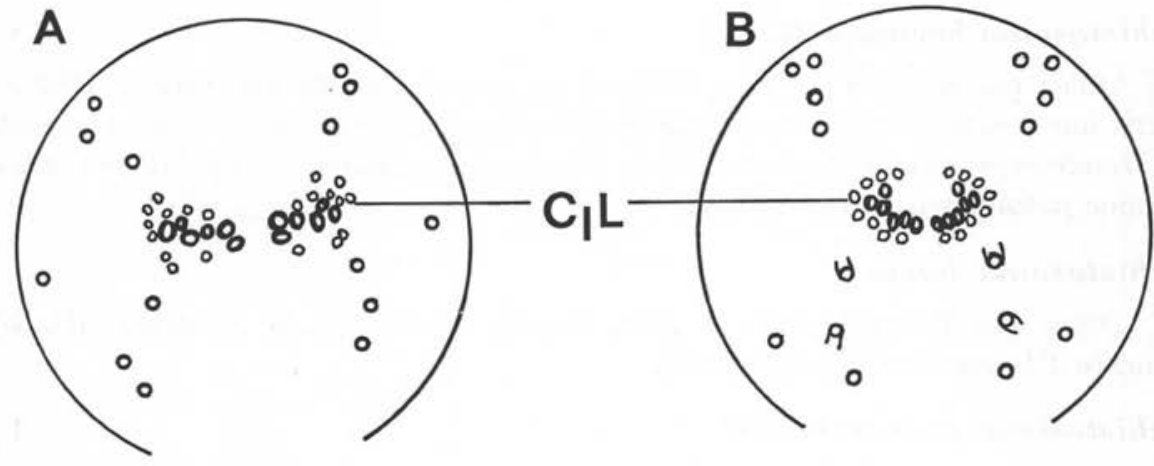

C
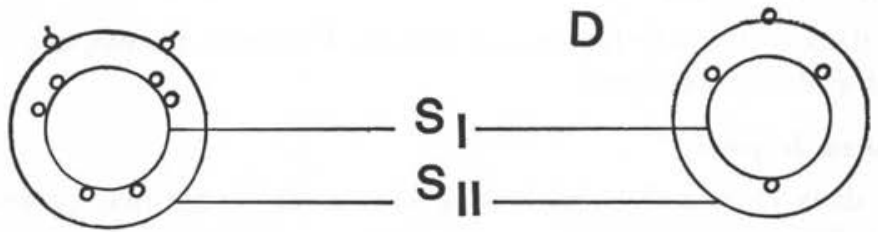

Fig. I. - A : Papilles céphaliques de Schistosomatium douthitti d'après Knos et Short (1979).

B : Papilles céphaliques de Schistosoma spp.

C : Papilles acétabulaires de Schistosomatium douthitti d'après Knos et Short (1979).

D : Papilles acétabulaires de Schistosoma spp.

Les papilles céphaliques (fig. $1 \mathrm{~A}$ )

Les papilles céphaliques $\mathrm{C}_{\mathrm{I}} \mathrm{L}$ sont au nombre de 10 de part et d'autre du plan médiosagittal chez Schistosomatium douthitti et de 6 à 8 chez Schistosoma spp.

Les papilles corporelles ventrales et dorsales (fig. $2 \mathrm{D}, 3 \mathrm{D}$ )

Ventralement, Schistosomatium douthitti est dépourvu de $A_{I} V$; Schistosoma spp a toujours une papille $\mathrm{A}_{\mathrm{I}} \mathrm{V}$. Dorsalement, Schistosomatium douthitti porte une papille $\mathrm{P}_{\mathrm{II}} \mathrm{D}$; Schistosoma spp en est toujours dépourvu.

Les papilles acétabulaires (fig. 1 C)

Les papilles acétabulaires sont au nombre de $6 \mathrm{~S}_{\mathrm{I}}$ et $2 \mathrm{~S}_{\mathrm{II}}$ chez Schistosomatium douthitti, de $3 \mathrm{~S}_{\mathrm{I}}$ et $1 \mathrm{~S}_{\mathrm{II}}$ chez Schistosoma spp. 
Les papilles caudales ventrales et dorsales (fig. 4 C)

Les papilles caudales ventrales et dorsales de Schistosomatium douthitti sont distribuées dans la moitié distale du tronc caudal; elles sont réparties tout au long du tronc caudal chez Schistosoma spp.

2) Caractéristiques de Schistosoma japonicum, parasite d'Oncomelania, Melaniidae (fig. $2 C, 3 C, 4 B_{\mathrm{V}} B_{\mathrm{D}}$ ).

Ces caractéristiques s'observent au niveau des papilles corporelles ventrales et dorsales et au niveau des papilles caudales de la cercaire.

Les papilles ventrales (fig. 2 C)

De part et d'autre du plan médio-sagittal, au nombre de 7 chez les deux souches décrites par Albaret et coll. (1982), au nombre de $8 \mathrm{chez}$ les Schistosomes de Bulininae et au nombre de 9 chez les Schistosomes de Planorbiinae.

Sakamoto et Ishii figurent 9 papilles chez la cercaire de S. japonicum, mais Albaret et coll. estiment que les auteurs japonais auraient confondu avec des papilles, des granulations situées en $\mathrm{A}_{\mathrm{II}} \mathrm{V}$ et $\mathrm{MV}$ et appelées V5 et V6 dans la description originale.

Les papilles dorsales ( fig. $3 \mathrm{C}$ )

$\mathrm{Au}$ nombre de $9 \mathrm{chez}$ Schistosoma japonicum avec une papille $\mathrm{A}_{\mathrm{IV}} \mathrm{D}$ et pas de papille MD; habituellement au nombre de $10 \mathrm{chez}$ les Schistosomes de Bulinidae avec $2 \mathrm{~A}_{\mathrm{III}} \mathrm{D}$ et $1 \mathrm{MD}$.

Les papilles caudales (fig. $4 \mathrm{BV}, \mathrm{BD}$ )

Peu nombreuses chez Schistosoma japonicum : 1 à 2 UL, 6 à 10 UV, 6 à 10 UD, 0 à 2 UF ; plus nombreuses chez les Schistosomes de Bulinidae qui ont 4 à 10 UL souvent 8,14 à $20 \mathrm{UV}, 14$ à $20 \mathrm{UD} ; 8$ à $16 \mathrm{UF}$ souvent 12.

3) Caractéristiques des Schistosomes de Planorbiinae Bulinidae : Schistosoma mansoni et $S$. rodhaini (fig. $2 \mathrm{~A}$ ).

Les cercaires de Schistosomes de Planorbiinae possèdent une papille $\mathrm{P}_{\mathrm{I}} \mathrm{V}$, soit un total de 9 papilles ventrales, de part et d'autre du plan médio-sagittal, les Schistosomes de Bulininae en ont 8.

4) Caractéristiques des Schistosomes de Bulininae Bulinidae : S. haematobium, S. bovis, S. margrebowiei, S. mattheei, S. leiperi et S. intercalatum (fig. $2 \mathrm{~B}$ ).

Les cercaires de Schistosomes de Bulininae sont dépourvus de papille $\mathrm{P}_{\mathrm{I}} \mathrm{V}$, elles possèdent 8 papilles ventrales de part et d'autre du plan médio-sagittal, elles se différencient des Schistosomes de Planorbiinae qui en ont 9. 

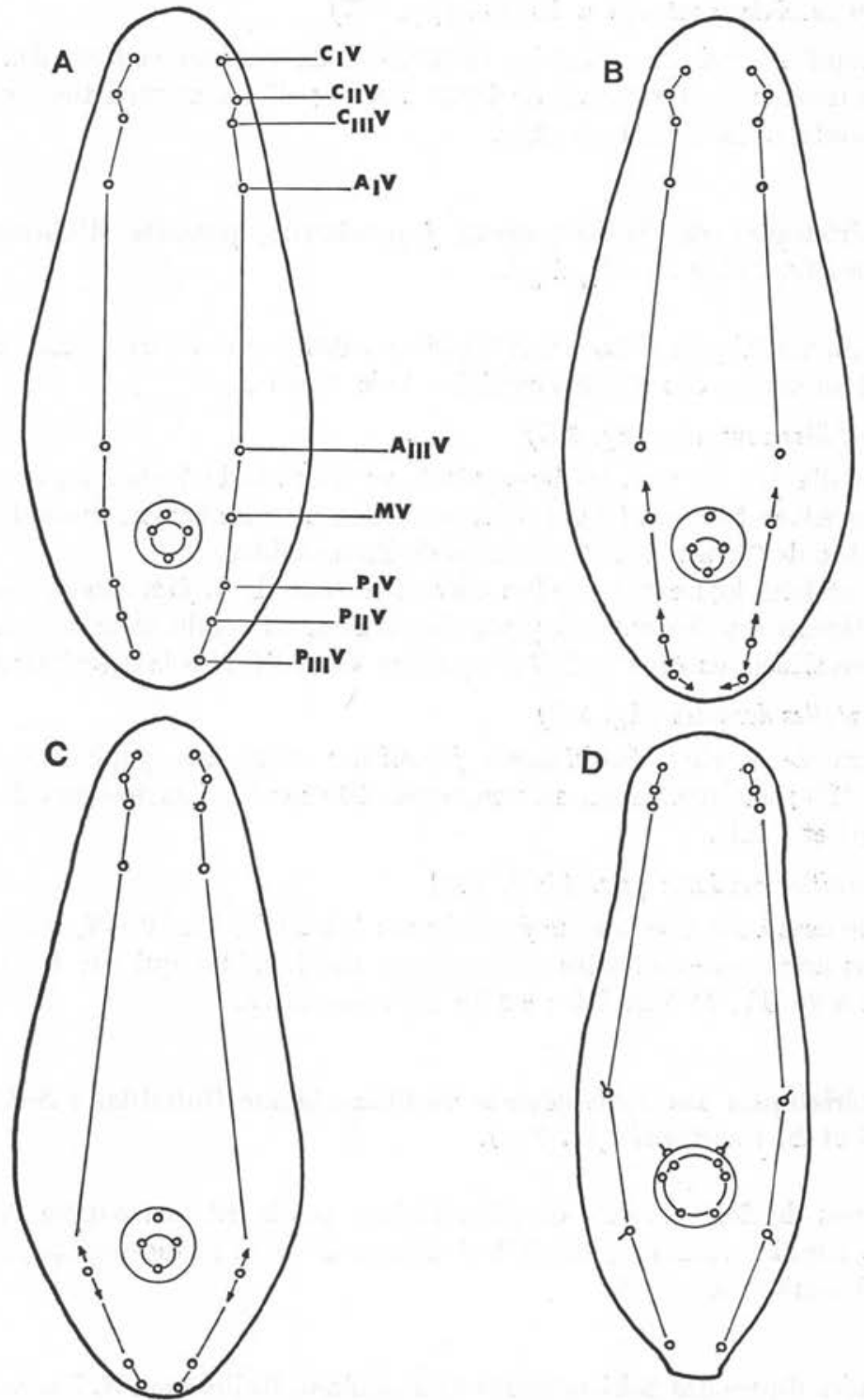

FIG. 2. - A : Papilles corporelles ventrales des Schistosomes de Planorbiinae.

B : Papilles corporelles ventrales des Schistosomes de Bulininae.

C : Papilles corporelles ventrales de Schistosoma japonicum d'après Albaret et coll. (1982).

D : Papilles corporelles ventrales de Schistosomatium douthitti d'après Knos et Short (1979).

Les papilles portant des flèches peuvent être situées antérieurement ou postérieurement à la position indiquée. 

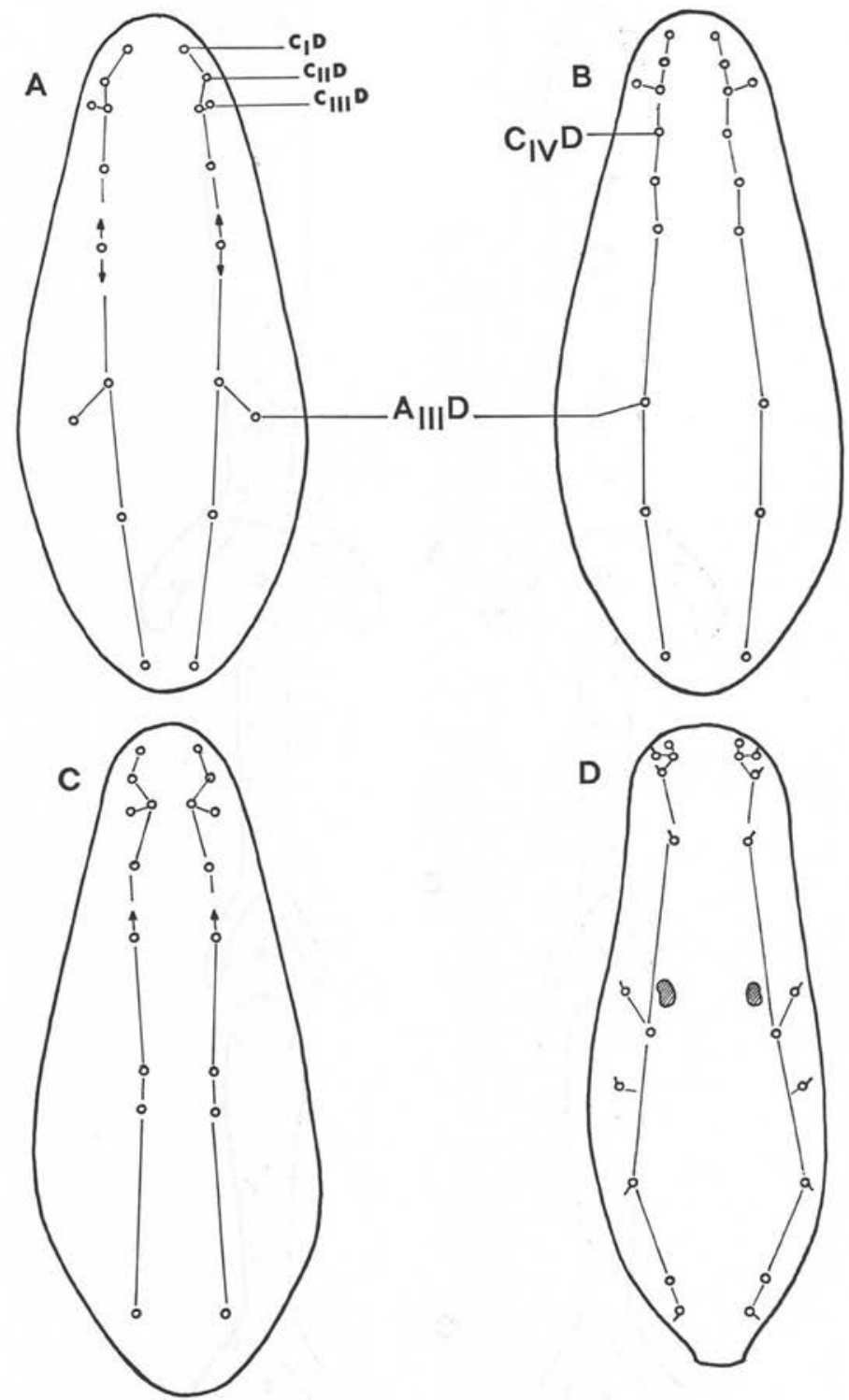

FIG. 3. - A : Papilles dorsales de S. mansoni et des Schistosomes de Bulininae.

B : Papilles dorsales de $S$, rodhaini.

C: Papilles dorsales de $S$, japonicum d'après Albaret et coll. (1982)

D : Papilles dorsales de Schistosomatium douthitti d'après Knos et Short (1979).

Les papilles portant des flèches peuvent être situées antérieurement ou postérieurement à la position indiquée. 

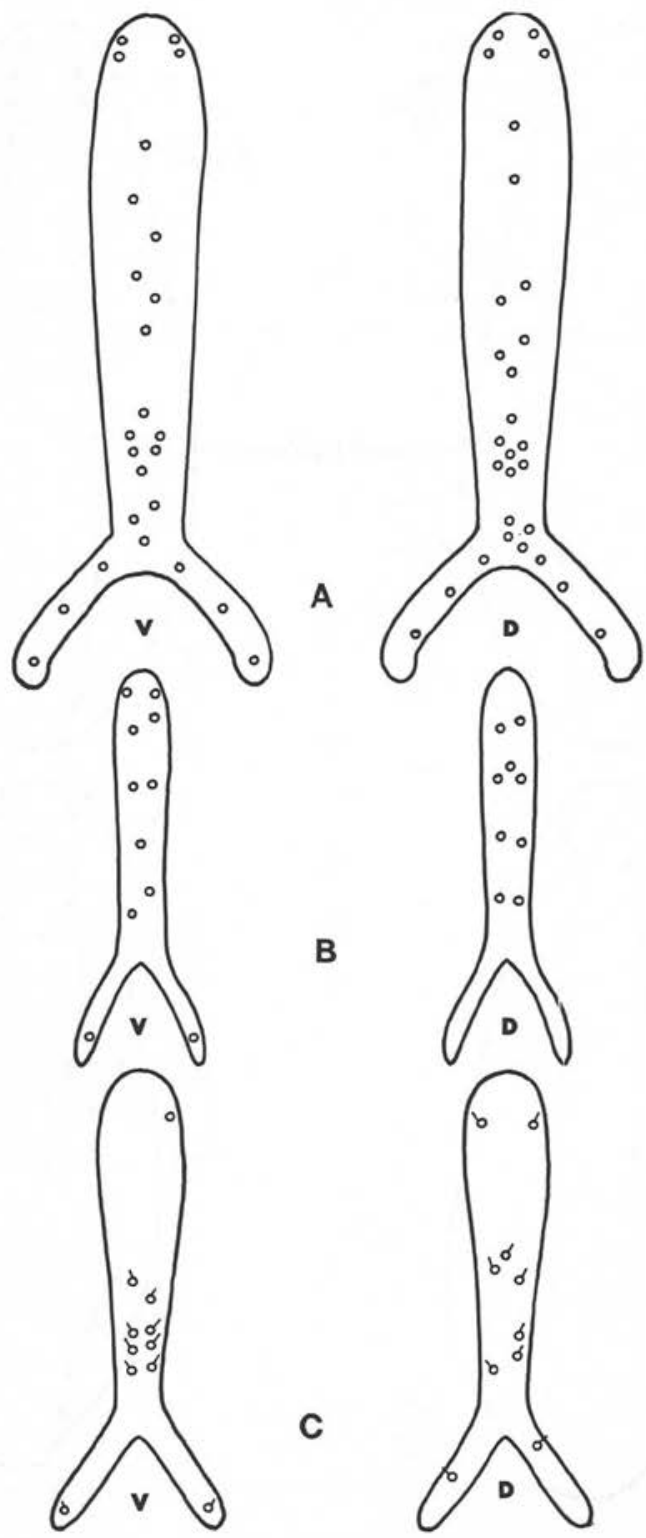

B

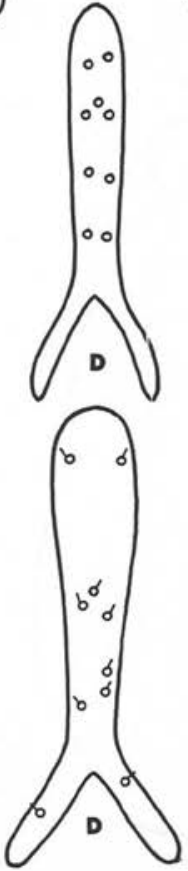

Fig. 4. - AV-AD : Papilles caudales ventrales et dorsales des Schistosomes de Bulinidae.

BV-BD : Papilles caudales ventrales et dorsales de Schistosoma japonicum d'après Albaret et coll. (1982).

CV-CD : Papilles caudales ventrales et dorsales de Schistosomatium douthitti d'après Knos et Short (I979). 


\section{III - Identification des deux espèces parasites de Planorbiinae : $S$. mansoni et $S$. rodhaini (fig. 3 A, B).}

La chétotaxie des papilles dorsales permet l'identification des deux espèces de Schistosomes parasites de Biomphalaria.

- S. mansoni a 10 papilles dorsales : 4 céphaliques, 6 corporelles dont $2 \mathrm{~A}_{\mathrm{III}} \mathrm{D}$.

$-S$. rodhaini a 10 papilles dorsales : 5 céphaliques dont $1 \mathrm{C}_{\mathrm{IV}} \mathrm{D}$ et 5 corporelles dont

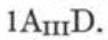

IV - Essai d'identification des espèces parasites de Bulininae : S. haematobium, S. bovis, S. margrebowiei, S. mattheei, S. leiperi et $S$. intercalatum (fig. 5 à 11 ).

Des différences topographiques discrètes existent à l'intérieur des souches considérées. Richard, (1971) utilisait deux caractères chétotaxiques pour reconnaître S. haematobium de $S$. bovis :

- l'écartement entre les papilles $\mathrm{A}_{\mathrm{I}} \mathrm{D}-\mathrm{A}_{\mathrm{II}} \mathrm{D}$ et $\mathrm{A}_{\mathrm{II}} \mathrm{D}-\mathrm{A}_{\mathrm{III}} \mathrm{D}$; caudal.

- la composition d'un groupe de papilles situées au quart postérieur du tronc

Pour essayer d'identifier les six espèces considérées, nous reprenons les deux caractères proposés par Richard et $\mathrm{y}$ ajoutons un troisième caractère : l'écartement entre les papilles $A_{I I I} V-M V$ et $P_{I I} V-P_{I I I V}$.

Nous obtenons alors des groupements d'espèces ;

1) L'écartement entre les papilles $A_{I} D-A_{I I} D$ et $A_{I I} D-A_{I I I} D$ soit $\frac{A_{I} D-A_{I I} D}{A_{I I} D-A_{I I I} D}=$ rapport $A_{D}$ permet la séparation en deux groupes :

a) le groupe $S$. haematobium, S. margrebowiei et $S$. mattheei chez lequel le rapport $A_{D}$ est inférieur ou égal à 1 ;

b) le groupe $S$. bovis, $S$. leiperi et $S$. intercalatum chez lequel le rapport $A_{D}$ est supérieur à 1 . (fig. $6,7,10,11$ )

2) La composition du groupe des papilles situées ventralement $U_{V}$ et dorsalement $U_{D}$ au quart postérieur du tronc caudal sépare en :

a) groupe $S$. haematobium et $S$. margrebowiei qui porte quatre papilles ventrales et quatre papilles dorsales ;

b) groupe $S$. bovis, S. mattheei, S. leiperi et $S$. intercalatum qui porte six papilles ventrales et six papilles dorsales. ( $f i g .8,9,10$ ) 


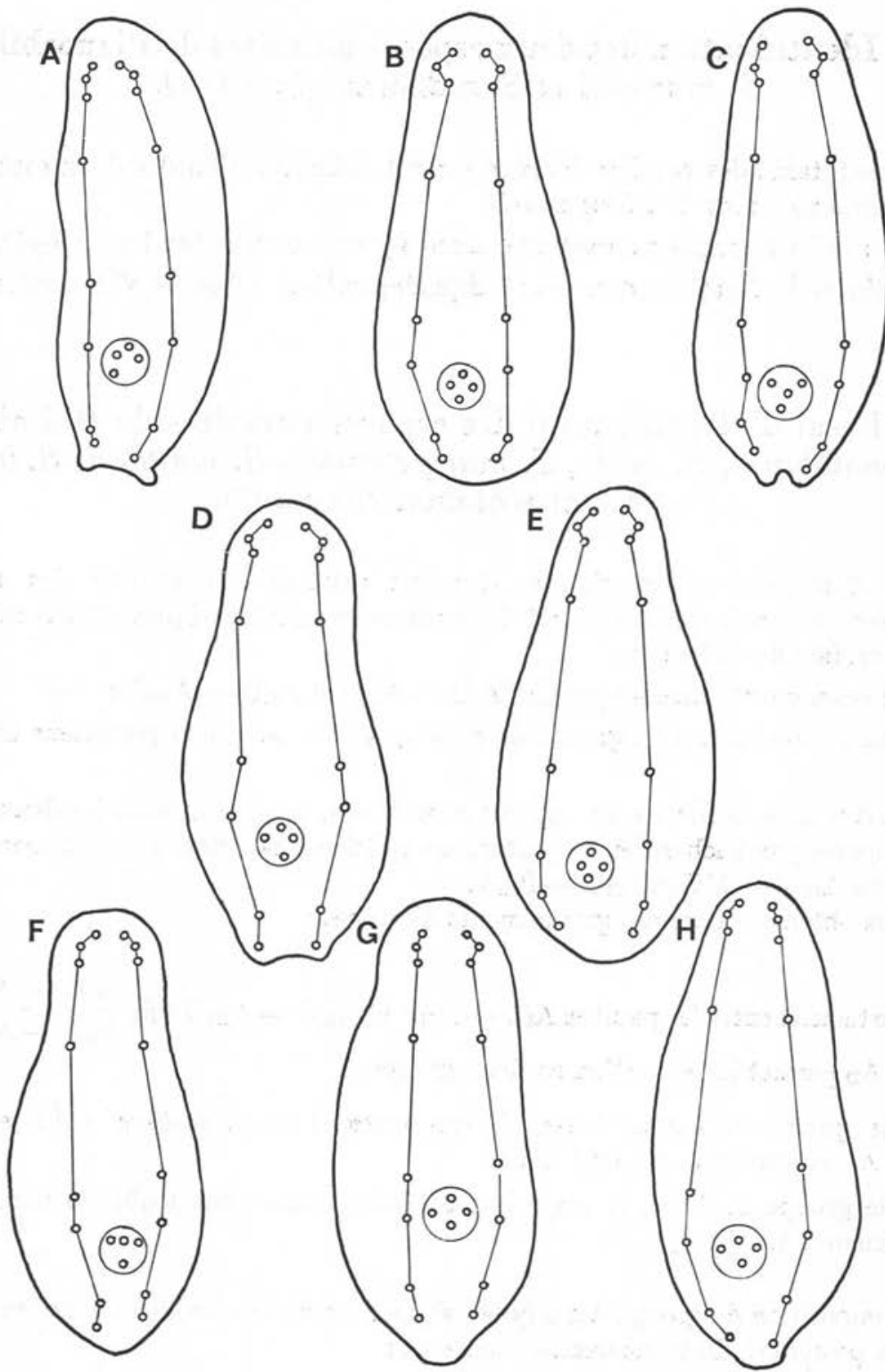

FIG. 5. - Papilles ventrales de 6 espèces de Schistosomes de Bulininae.

A : S. haematobium d'après Richard (1971).

C : S. bovis d'après Richard (197I).

G : $S$. intercalatum d'après Bayssade-Dufour et coll. (1980).

B : S. haematobium ; D : S. margrebowiei; E : S. mattheei.

$\mathrm{F}: S$. leiperi ; H : S. intercalatum; descriptions du présent travail. 


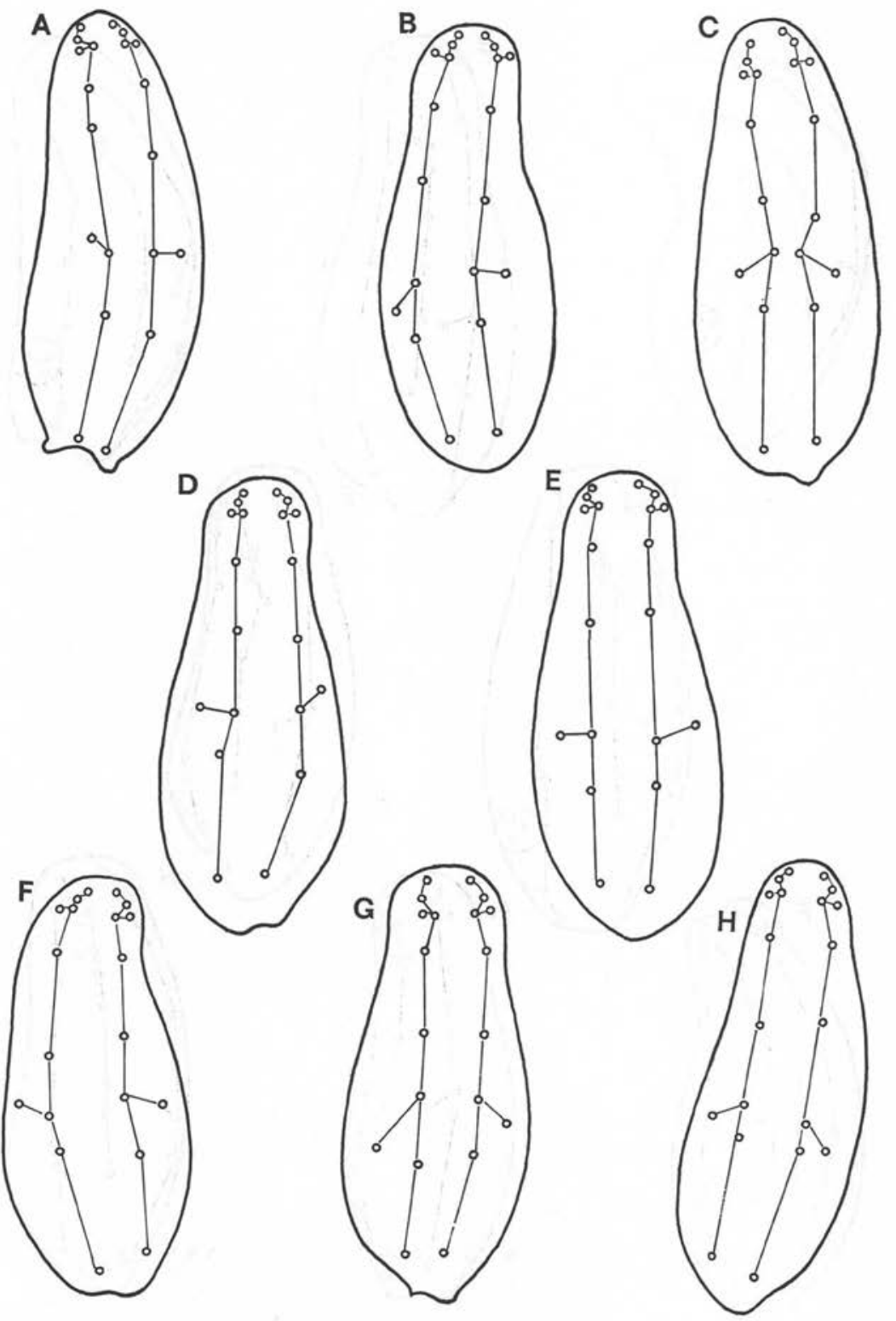

Fig. 6. - Papilles dorsales de 6 espèces de Schistosomes de Bulininae.

A : S. haematobium d'après Richard (197I).

C: $S$. bovis d'après Richard (1971).

G : S. intercalatum d'après Bayssade-Dufour et coll. (I980).

$\mathrm{B}: S$. haematobium; $\mathrm{D}: S$. margrebowiei; $\mathrm{E}: S$. mattheei.

$\mathrm{F}: S$. leiperi ; H : $S$. intercalatum ; descriptions du présent travail. 

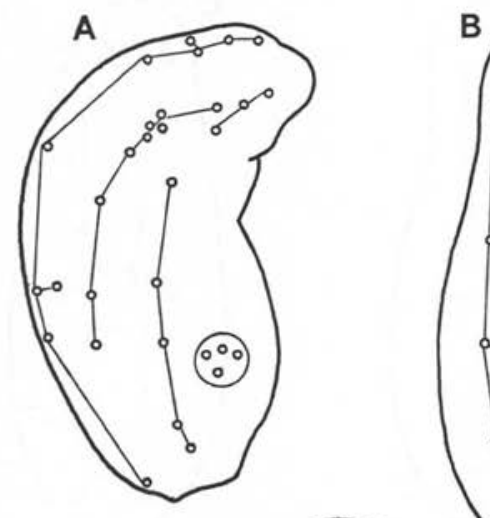

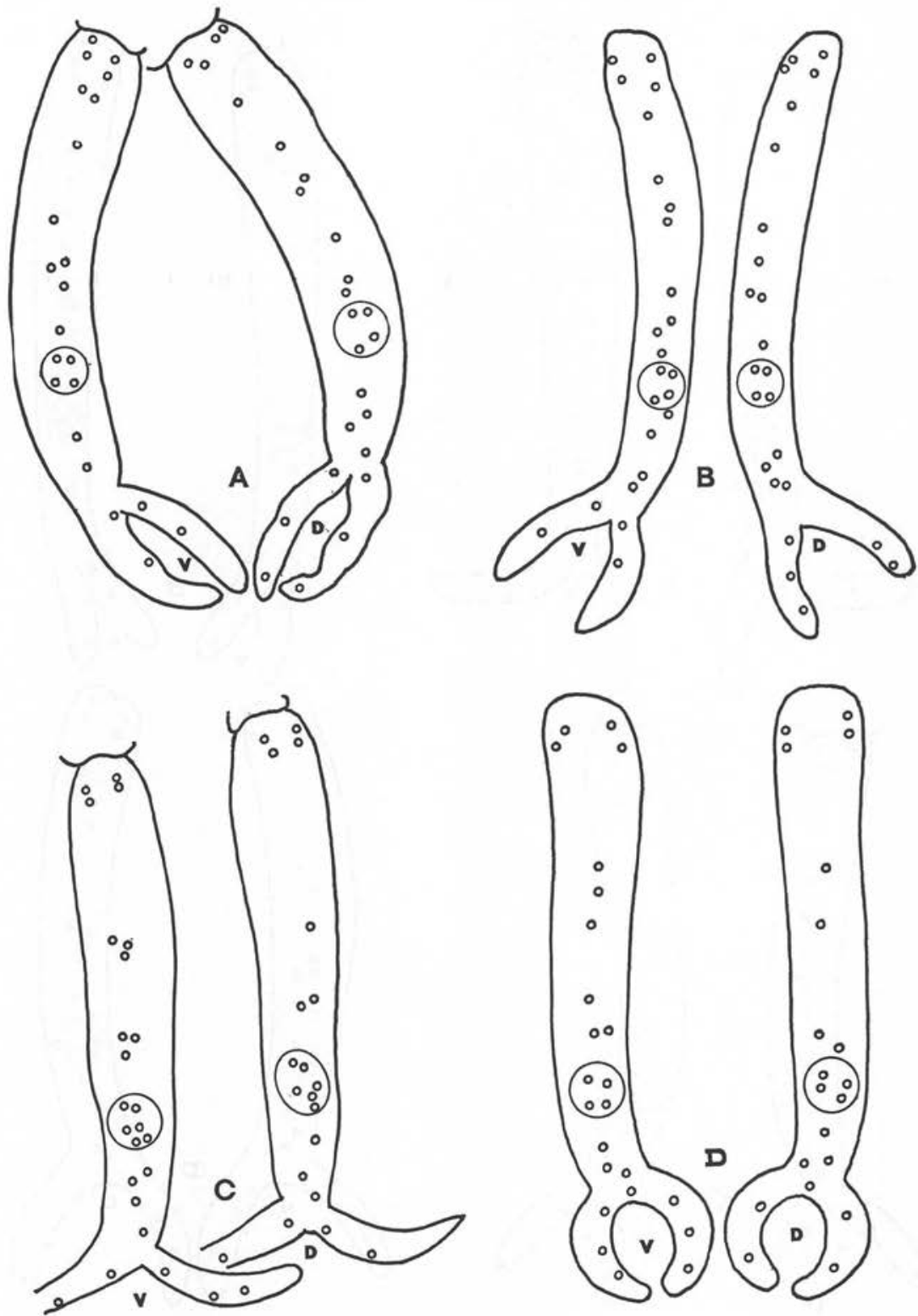

FIG. 8. - Papilles caudales ventrales (V) et dorsales (D) de Schistosomes de Bulininae.

A : S. haematobium d'après Richard (197I).

C : S. bovis d'après Richard (1971).

B : S. haematobium; D : S. margrebowiei; descriptions du présent travail. 

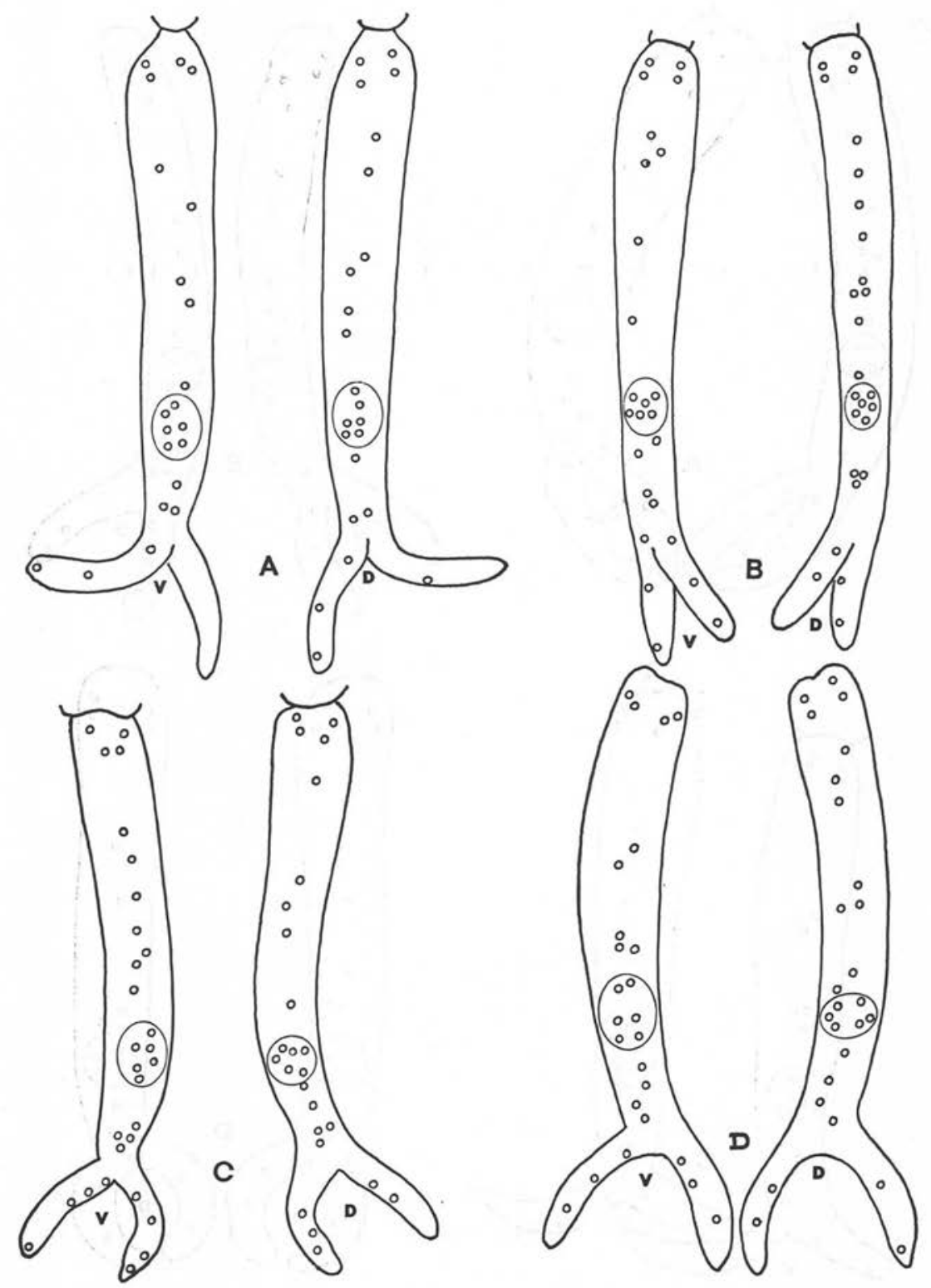

FIG. 9. - Papilles caudales ventrales (V) et dorsales (D) de Schistosomes de Bulininae. C : S. intercalatum d'après Bayssade-Dufour et coll. (I980).

A :S. mattheei ; B : S. leiperi ; D : S. intercalatum; descriptions du présent travail. 


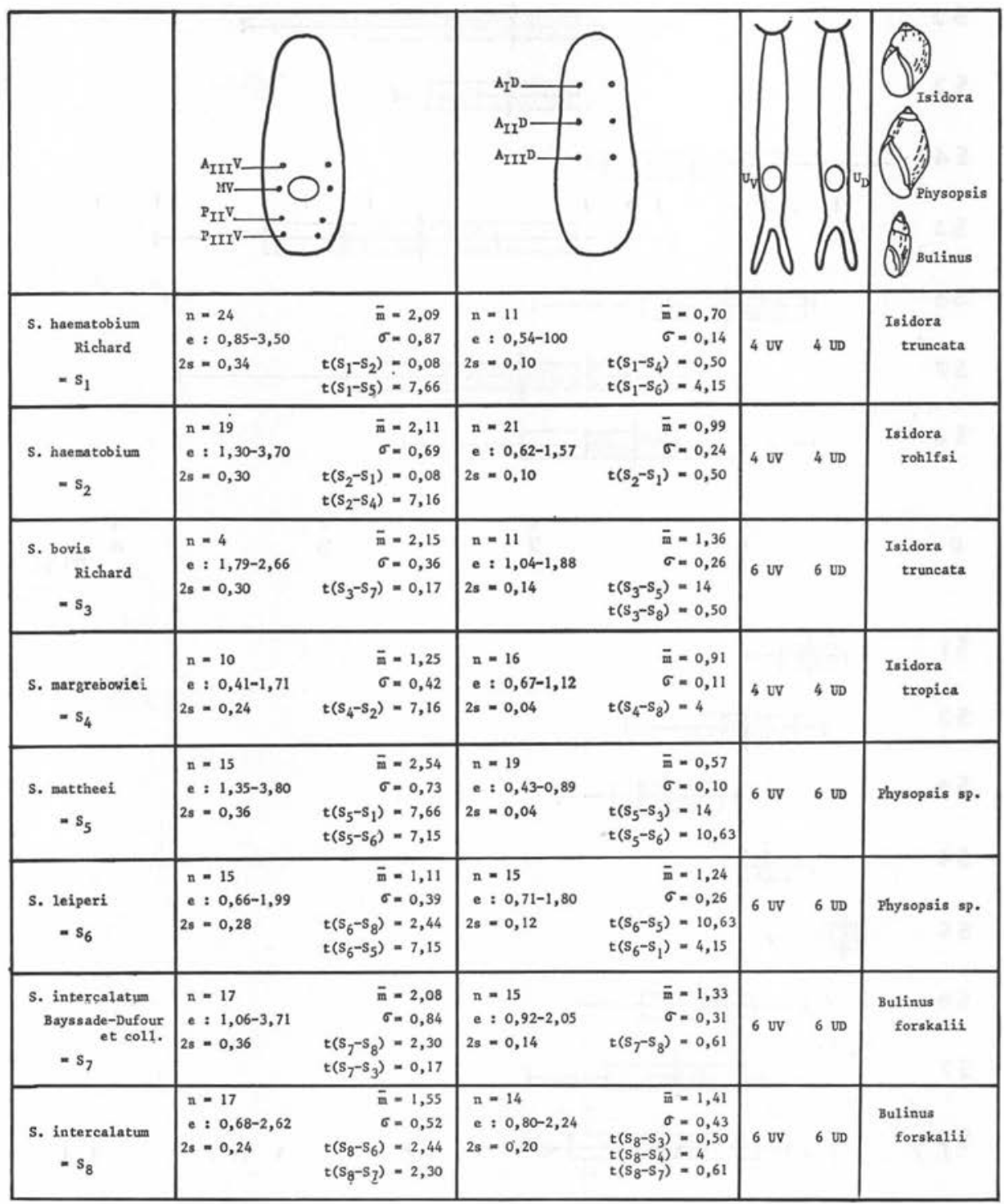

FIG. ro. - Tableau récapitulant les caractéristiques des Schistosomes de Bulininae ; n : nombre d'échantillons, m : moyenne de l'échantillon; e : extrêmes observés ; $\sigma$ : écart-type ; $2 \mathrm{~S}$ : intervalle de confiance de la moyenne ; $t$ : écart-réduit ; les valeurs de $t$ inférieures ou égales à 2,0 sont non significatives. 
S 1

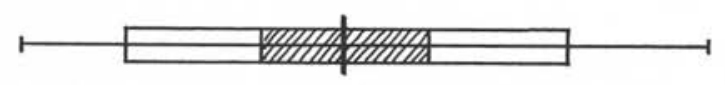

S 2

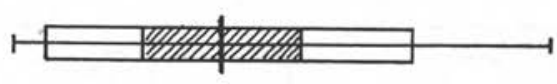

S 3

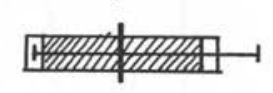

54

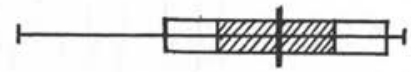

S 5

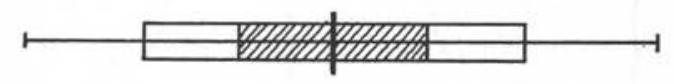

56

S 7

S 8

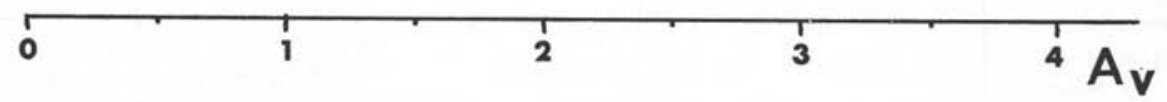

S1

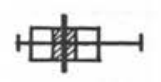

52

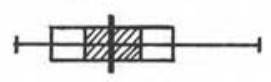

S3

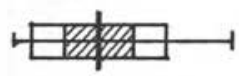

54

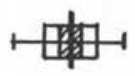

S5

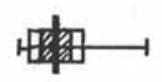

56

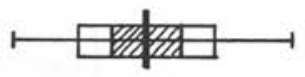

S7

S 8
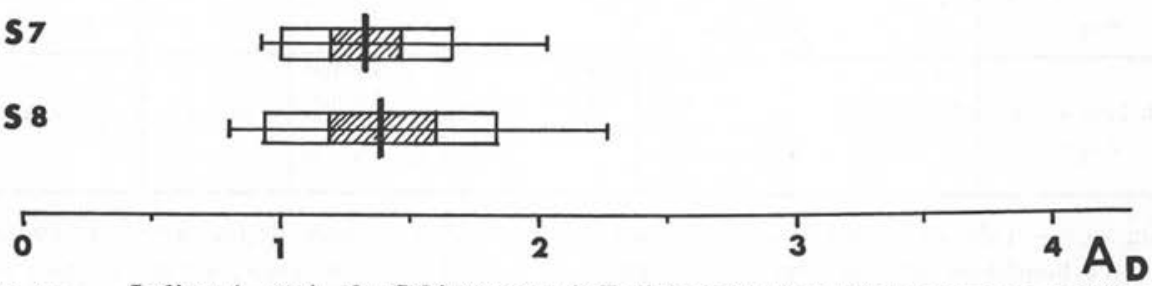

FIG. II. - Indices $A_{y}$ et $A_{D}$ des Schistosomes de Bulininae. Trait vertical : moyenne de l'échantillon; rectangle hachuré : intervalle de confiance de la moyenne; rectangle blanc : écarttype de l'échantillon; ligne horizontale : extrême observées. Pour l'identification des différentes souches $\mathrm{S}$, voir fig. Io. 
3) L'écartement entre les papilles $A_{I I I} V-M V$ et $P_{I I} V-P_{I I I} V$ soit $\frac{A_{I I I} V-M V}{P_{I I} V-P_{I I I} V}=$ rapport $A_{V}$ sépare en :

a) groupe $S$. margrebowiei et $S$. leiperi chez lequel le rapport $A_{v}$ est légèrement supérieur à 1 ;

b) groupe $S$. haematobium, S. bovis, S. mattheei et $S$. intercalatum chez lequel le rapport $A_{V}$ est compris entre 1,5 et 3. (fig. $5,10,11$ )

\section{Discussion}

Il est difficile d'apprécier la valeur des caractères que nous venons de mettre en évidence car nous.ne disposons, le plus souvent, que d'une à deux souches par espèce ; parfois nous analysons des souches de laboratoire entretenues par passages successifs sur des hôtes expérimentaux et qui peuvent différer, dans une certaine mesure des espèces naturelles; cf. Cassone et coll. (1978).

Ces réserves étant faites, le calcul, pour chaque souche, des indices $A_{V}$ et $A_{D}$, leur moyenne $\overline{\mathrm{m}}$ pour un échantillon $\mathrm{n}$, leur intervalle de confiance 2 s, leur écart-type $\sigma$ et leur écart-réduit t permet de comparer deux à deux les moyennes (cf. Schwartz 1977, p. 158) et d'observer les résultats suivants :

a) S. haematobium du Maroc entretenue sur Isidora truncata ne diffère pas significativement de $S$. haematobium de RCA passée sur Isidora rohlfsi.

L'indice $A_{D}$ est assez différent mais nous ne disposons que de 11 mesures pour la souche marocaine de $S$. haematobium.

b) S. margrebowiei, du fait de l'indice $\mathrm{U}_{\mathrm{V}} \mathrm{U}_{\mathrm{D}}$ ne peut être rapproché que de $S$. haematobium; toutefois l'indice $A_{\mathrm{v}}$ en diffère significativement, selon le test de l'écart-réduit et nous considérons $S$. margrebowiei comme une bonne espèce.

c) Nos deux souches de $S$. intercalatum, bien que toutes deux d'origine gabonaise et toutes deux passées une fois sur Bulinus forskalii, diffèrent significativement en ce qui concerne l'indice $A_{V}$; nous ne comprenons pas la signification de cette différence.

d) S. bovis, issu d'un Mouton de Sardaigne et conservé en laboratoire par passage chez Isidora truncata ne diffère pas de S. intercalatum; notre échantillonage de $S$. bovis est faible mais les données dont nous disposons pourraient s'interpréter par la synonymie des deux espèces.

e) S. leiperi du Botswana entretenu sur Physopsis sp. et Praomys natalensis $a$ un indice $A_{D}$ qui diffère peu, mais de façon significative d'une des deux souches de S. intercalatum. $\left(\mathrm{A}_{\mathrm{D}}=1,55\right)$; là encore l'individualité de l'espèce est vraisemblable.

f) S. mattheei d'Afrique du Sud entretenue sur Physopsis sp et Praomys natalensis se comporte également comme une espèce nettement originale, son indice $A_{V}$ étant très élevé et son indice $A_{D}$ très faible. 


\section{Conclusion}

Dans l'état actuel de nos recherches, nous différencions bien par la chétotaxie le genre Schistosomatium du genre Schistosoma, les Schistosoma de Melaniidae des Schistosoma de Bulinidae, les deux espèces de Schistosoma, parasites de Planorbiinae des Schistosoma, parasites de Bulininae. Au contraire, nous différencions mal, les unes des autres, les différentes espèces de Schistosomes de Bulininae. Certes, nos données comportent des sources d'erreur car nous ne pouvons comparer que quelques souches particulières pour chaque espèce, et nos données numériques sont parfois trop peu abondantes : ainsi $S$. haematobium de RCA est différent de celui du Maroc, mais avec les données dont nous disposons, la différence n'est pas significative; $S$. margrebowiei et $S$. leiperi se comportent toutes deux comme de bonnes espèces. Nous avons au Gabon deux souches de $S$. intercalatum qui montrent une différence significative, par contre $S$. bovis ne se sépare pas de l'une de ces deux souches de S. intercalatum.

Par ailleurs, Schistosoma japonicum qui porte 6 axes caudaux de papilles : 2 ventraux, 2 dorsaux et 2 latéraux serait plus évolué que les Schistosoma de Bulinidae qui en portent $8: 2$ ventraux, 2 dorsaux et 4 latéraux; mais il serait plus primitif que les Bilharzies d'Oiseaux qui en portent 4: Trichobilharzia ocellata, T. brevis, Austrobilharzia terrigalensis, Ornithobilharzia canaliculata, cf. Richard (1971), Bayssade-Dufour et Ow-Yang (1975), Rohde (1977), Wagner (1961). Ces résultats sont en accord avec ceux d'Albaret (1982) qui conclut à l'existence de trois groupes de miracidiums de Schistosoma:

- le groupe $S$. mansoni auquel se rattache $S$. rodhaini;

- le groupe très homogène des Schistosoma de Bulininae ;

- et le groupe $S$. japonicum, très différent des deux premiers, mais présentant des affinités avec ceux de Schistosomatium et des Bilharzies d'Oiseaux.

Nous adressons nos plus vifs remerciements au Dr. R. J. Pitchford qui nous a procuré quatre souches de Schistosomes : S. rodhaini, S. margrebowiei, S. mattheei et $S$. leiperi.

Une des souches de Schistosoma japonicum décrite en collaboration avec Albaret et Tsuji a été fournie par l'Université de Lowel, Massachusetts 01854, contrat AI 72524.

\section{BIBLIOGRAPHIE}

Albaret J. L. : Comparaison des organites argyrophiles des miracidiums de Schistosomatidae, I982 (en préparation)

Albaret J. L., BAYSSADE-Dufour Ch., Tsujr M. : Les structures argyrophiles superficielles des formes larvaires infestantes de Schistosoma japonicum. Ann. Parasitol. Hum. Comp., 1982 (à paraitre).

BAYSSADE-DUfour Ch. : Différences au niveau des sensilles entre les cercaires africaines et américaines de Schistosoma mansoni, agent de la bilharziose intestinale humaine. C. R. Acad. Sci., Paris, 1977 a, 284 , sér. D, I9I-193. 
BAYSSADE-DUfour Ch. : Variations chétotaxiques chez les cercaires de Schistosoma mansoni, agent de la bilharziose intestinale, en corrélation avec l'hôte vertébré du parasite. C. R. Acad., Sci., Paris 1977 b, 285 , sér. D, I5II-1513.

BAYSSADE-DUFOUR Ch. : Variation du système sensoriel de la cercaire de Schistosoma mansoni. Intérêt éventuel en épidémiologie. Ann. Parasitol. Hum. Comp., I979, 54, 596-6r4.

Bayssade-Dufour Ch., Albaret J. L., Picot H., Deniau M. : Les organites argyrophiles superficiels des formes larvaires infestantes de Schistosoma intercalatum. Ann. Parasitol. Hum. Comp., 1980, s5, 249-252.

BAYSSADE-DUFOUR Ch., OW-Y ANG C. K. : The sensory receptors of two cercariae from Malaysia : Trichobilharzia brevis (Schistosomatidae) and Haplorchis pumilio (Heterophyidae). Southeast Asian. J. Trop. Med. Pub. health, 1975, 6, 338-342.

CAssone J. : Variations chétotaxiques des cercaires d'une souche humaine de Schistosoma mansoni par passages successifs sur Souris. Ann. Parasitol. Hum. Comp., 1978, 53, 387-391.

FrIPP P. J. : On the morphology of Schistosoma rodhaini (Trematoda, Digenea, Schistosomatidae). J. Zool. London, I967, ISI, 433-452.

KNos G. B., SHORT R. B. : Argentophilic papillae of Schistosomatium douthitti cercariae (Trematoda, Schistosomatidae). J. Parasitol., 1979, 65, 350-356.

Richard J. : La chétotaxie des cercaires de Schistosomes. C. R. Acad. Sc., Paris, 1968, 266. sér. D, I $856-1859$.

RICHARD J. : La chétotaxie des cercaires. Valeur systématique et phylétique. Mém. Mus. Nat. Hist. Nat., 1971, sér. A Zool., 67, 1-179.

RoHDE K. : The bird Schistosome Austrobilharzia terrigalensis from the Great Barrier Reef, Australia. Z. Parasitkde, 1977, 52, 39-51.

SAKAмото K., IsHII Y. : Scanning electron microscope observations on miracidium, cercaria and cercarial papillar patterns of Schistosoma japonicum. J. Parasitol., 1978, 64, 59-68.

SchWARTZ D. : Méthodes statistiques à l'usage des médecins et des biologistes, 318 p., Flammarion, Paris, 1977 .

Short R. B., CARtrett M. L. : Argentophilic papillae of Schistosoma mansoni cercariae. J. Parasitol., 1973, 59, 104I-1059.

SHORT R. B., KUNTZ M. L. : Patterns of argentophilic papillae of Schistosoma rodhaini and S. mansoni cercariae. J. Parasitol., 1976, 62, 420-425.

Vercammen-Grandjean P. H. : Sur la chétotaxie de la larve infestante de $S$. mansoni. Ann. Parasitol. Hum. Comp., 1951, 26, 4I2-4I4.

WAGNer A. : Papillae on three species of Schistosoma cercariae. J. Parasitol., 1961, 47, 614-618. 